\title{
Evaluation of Effect Assessment on Case-Management of Women with Gestational Diabetes Mellitus
}

\author{
Huang Xinke*, Huang Dongxi, Feng Nan \\ Neoatal Department, The First Affiliated Hospital of Jinan University, Guangzhou, China \\ Email address: \\ 929901838@qq.com (Huang Xinke),874845715@qq.com (Huang Dongxi), 1158192234@qq.com (Feng Nan) \\ *Corresponding author
}

To cite this article:

Huang Xinke, Huang Dongxi, Feng Nan. Evaluation of Effect Assessment on Case-Management of Women with Gestational Diabetes Mellitus. American Journal of Nursing Science. Vol. 8, No. 6, 2019, pp. 313-316. doi: 10.11648/j.ajns.20190806.14

Received: August 26, 2019; Accepted: September 24, 2019; Published: October 23, 2019

\begin{abstract}
Explore the application of case management in gestational diabetes mellitus (GDM). 164 patients diagnosed as GDM from February to November 2016 were set up as control group. 196 patients diagnosed as GDM from February to November of 2017 were divided into intervention group. In the control group, gynecology and obstetrics clinic doctors and nurses directly carried out routine health education, diet guidance and exercise advice. The intervention group adopted the case management model of gestational diabetes mellitus, including six major modules of case selection, overall service needs assessment, goal-oriented case management plan, follow-up management, contact and coordination to obtain services and evaluate case success, GDM women for blood glucose management, comparison of case management before and after GDM patients, the difference between the outcome of pregnancy. GDM case management model could effectively control the blood glucose of patients with GDM, reduce the readmission rate due to poor blood glucose control $(P<0.001)$, and significantly reduce the insulin used rate of patients with $\operatorname{GDM}(P<0.019)$, but there was no significant difference between the two groups of the outcome of pregnancy $(P>0.05)$. Case management of gestational diabetes mellitus can effectively save medical costs and play an important role in the management of women with GDM.
\end{abstract}

Keywords: Case Management, Gestational Diabetes Mellitus, Effectiveness Evaluation

\section{Introduction}

Gestational diabetes mellitus (GDM) is defined that the first abnormality of glucose metabolism during pregnancy $[1,14]$. The incidence of GDM is increasing as the quality of life is improved, GDM not only make damage to the body of pregnant woman, but also increase the budget of the economy and mental burden, that they increase the risk of perinatal complications such as macrosomia, neonatal hypoglycemia, fetal respiratory distress and stillbirth [2]. So active nursing intervention is of great significance to improve outcomes of maternal and infant. Case management is a mode of systematically providing care services according to the disease characteristics, individual needs and the application what is combines medical technology and communication [3]. Nowadays, case management model has been widely used in the field of nursing. This study aims to use case management model to conduct blood glucose management in the patients that has GDM, and to evaluate the impact of case management model on blood glucose control and maternal and infant outcomes.

\section{Samples and Methods}

\subsection{Patients Enrollment}

Based on convenience sampling, we investigate the patients that were diagnosed with GDM, that patients with GDM who their diagnosis of time from February 2016 to November 2016 was included in the control group and patients with GDM who their diagnosis of time from February 2017 to November 2017 was included in the experimental group. Patients were eligible for enrollment in the study if they: (1) was diagnosed with GDM according to Guidelines for Diagnosis and Treatment of Gestational Diabetes Mellitus (2014) [1]; (2) build the account of labor inspection in the First Affiliated Hospital of Jinan University and labor inspection in the hospital; (3) have the plan to childbirth in the hospital; (4) agreed to participate 
in this study. Withdraw criteria were: (1) have serious complications during pregnancy; (2) multiple pregnancies. For one thing, the control group was studied by medical record review; for other things, the intervention group was studied by follow-up from admission to discharge.

\subsection{Survey Methods}

The control group: doctors and nurses provide health education, dietary guidance and exercise advice on a regular basis.

The intervention group: the case management process of GDM patients was developed by nurses with public nutritionists and health management experts as case managers. The details of the case management process to include: firstly, case of screening: sending patient records from outpatient to case manager; secondly, evaluating the patient holistically: (1) Knowledge of GDM, (2) Evaluate the eating habits and the cooking style by 24-hour dietary review survey and NQA-PI individual nutrition analyzer, (3) The exercise mode and the amount of exercise what are suitable for the current body of patients, (4) Prepregnancy weight status and weekly weight gain during pregnancy, (5) Work and rest time and sleep quality, (6) patients's views and reactions to the diseases, and confidence in self-management of blood sugar; thirdly, guiding patients: the staff to carry out individual knowledge dissemination and make self-management plan for patients according to knowledge situation of patients, and the staff encourages family members to participate in the management of blood glucose in patients; fourthly, follow up patient status on time: weekly follow-up of blood glucose management in patients after the pregnant woman who were start self-management blood sugar has gone home, and providing the guide to patients according to their management problems of blood sugar. In detail, the patients were divided into two levels such as WhiteA1 Level and WhiteA2 Level, that they are managed separately. The patients of WhiteA1 Level were recorded as blood sugar control and weight to gain, also, the monitoring was continued when control is good and staff would solve the problems of patients in blood glucose management process. On the basis of WhiteA1 Level, staff would record the insulin injection of patients such as blood glucose control, hypoglycemia, needle renew, rotation of injection site and induration at the injection site; fifthly, coordinate contact: staff would ask patients return to the hospital in time and the doctor in charge was contacted to discuss the next diagnosis and treatment plan for patients, when the patients have poor blood sugar control and they can not solve problems by themselves. Moreover, consultation obstetrics and endocrinology was conducted for abnormal blood glucose in patients by multidisciplinary, while it not only improves communication between patients and staffs but also strengthen the diagnosis and treatment of patients. After that, the cases would be finished from 6 to 12 weeks postpartum of patients; finally, evaluation of case effectiveness: staffs recorded pregnancy outcomes, weight gain during pregnancy, insulin use, and readmission with poor blood sugar control were enrolled.

The evaluation index of patients and neonatal evaluation index use different evaluation methods. In the evaluation index of patients, they are include that the readmission rate, insulin utilization rate, cesarean section rate, postpartum hemorrhage rate and gestational period of patients with poor blood glucose control. In neonatal evaluation index, they are contains that Neonatal birth weight, incidence of macrosomia, incidence of premature infants, incidence of neonatal hypoglycemia, incidence of neonatal asphyxia and transfer ratio to neonatology department.

\subsection{Statistical Analysis}

Statistical analysis was performed using SPSS24.0. Data onto qualitative variables were reported as percentage (categorical data) and mean standard deviation (measurement data) for statistical description. Also, the two groups of data were compared and analyzed by t test for two independent samples, and comparative analysis of two groups was conducted by chi-square test.

\section{Result}

\subsection{Comparison of General Data}

There was no significant difference between the control group and the intervention group in age, OGTT value, ratio of first birth/transparturient $(\mathrm{P}>0.05)$ (Table 1). In the control group, the information include that 164 patients, aged (32.65 \pm 4.71$)$ years old, had fasting blood glucose $(4.45 \pm 0.65)$ $\mathrm{mmol} / \mathrm{L}, 1$ hour after sugar administration (9.80 \pm 1.65$)$ $\mathrm{mmol} / \mathrm{L}$, and 2 hours after sugar administration $(8.73 \pm 1.54)$ $\mathrm{mmol} / \mathrm{L}$, they include 40 first-time mothers and 124 multipara. In the intervention group, the information contains 196 patients, aged $(32.39 \pm 4.77)$ years old, had fasting blood glucose $(4.56 \pm 0.66) \mathrm{mmol} / \mathrm{L}, 1$ hour after sugar administration $(10.39 \pm 1.50) \mathrm{mmol} / \mathrm{L}, 2$ hours after sugar administration $(8.99 \pm 1.36) \mathrm{mmol} / \mathrm{L}$, they include 65 first-time mothers and 131 multipara.

Table 1. Comparison of general data between two groups.

\begin{tabular}{|c|c|c|c|c|c|}
\hline & Numbers of cases & Age & Weight & OGTT value & $\begin{array}{l}\text { Ratio of first } \\
\text { birth/Transparturient }\end{array}$ \\
\hline Control Group & 164 & $32.24 \pm 4.88$ years old & $45.62 \pm 7.55 \mathrm{KG}$ & $8.73 \pm 1.54$ & $24.39 \% / 66.84 \%$ \\
\hline Intervention Group & 196 & $32.24 \pm 4.88$ years old & $49.33 \pm 6.24 \mathrm{KG}$ & $8.99 \pm 1.36$ & $33.16 \% / 34.8 \%$ \\
\hline$P$ & - & 0.671 & 0.183 & 0.023 & - \\
\hline
\end{tabular}




\subsection{Comparison of Blood Glucose Control in Patients During Pregnancy}

In terms of the proportion of insulin use and the readmission rate of patients with poor blood glucose control, the intervention group was less than the control group, and the difference was statistically significant (Table 2).

\subsection{Comparison of Pregnancy Outcomes Among Patients}

There were no statistically significant differences between the two groups in cesarean section, gestational week of delivery, newborn birth weight, neonatal transfer, macrosomia, premature infants, neonatal hypoglycemia and et al. (Table 2).

Table 2. Comparison of Maternal and Neonatal Outcomes between the two groups [cases (percentage, \%)].

\begin{tabular}{|c|c|c|c|c|}
\hline Project & Intervention group $(n=164)$ & Control group $(n=196)$ & Statistic & $P$ Value \\
\hline Cesarean Delivery & $65(39.6)$ & $65(33.2)$ & 2.651 & 0.103 \\
\hline Poor Blood Glucose Control and Readmission & $6(3.7)$ & $31(15.8)$ & 24.826 & $<0.001$ \\
\hline Use Insulin & $36(22.0)$ & $24(12.4)$ & 5.474 & 0.019 \\
\hline Delivery Gestational Age & $38.94 \pm 1.23$ & $38.32 \pm 1.22$ & 0.980 & 0.328 \\
\hline Whether to Transfer to the Neonatal Department & $53(52.3)$ & $20(10.2)$ & 0.421 & 0.517 \\
\hline Neonatal Birth Weight & $3.25 \pm 0.47$ & $3.11 \pm 0.41$ & 0.339 & 0.735 \\
\hline Macrosomia & $1(0.6)$ & $2(1.0)$ & 2.897 & 0.089 \\
\hline Neonatal Hypoglycemia & $0(0)$ & $0(0)$ & - & - \\
\hline Neonatal Asphyxia & $1(0.6)$ & $2(1.0)$ & - & 1.00 \\
\hline Good Quality of Life & $77(47.0)$ & $93(47.4)$ & - & 0.027 \\
\hline Hypoglycemia & $32(19.5)$ & $12(6.1)$ & - & 0.041 \\
\hline Depression & $28(17.1)$ & $17(8.7)$ & - & 0.044 \\
\hline Anxiety & $25(15.2)$ & $16(8.2)$ & - & 0.022 \\
\hline
\end{tabular}

\section{Discussion}

\subsection{The Role of Case Management Model in Blood Glucose Management During Pregnancy}

The results of this study show that the application of case management model in patients with gestational diabetes is better than the traditional nursing model, which can reduce the utilization rate of insulin and readmission rate of patients with poor blood glucose control, which is consistent with the research results of $\mathrm{Li} \mathrm{Li}$ [4]. Case management provide integrated nursing services for patients according to the characteristics of the disease and the needs of patients, through effective communication, coordination and reasonable allocation of resources. The report shown that the case management model has been used in the whole nursing process of diabetic patients, and remarkable results have been achieved in improving medical treatment and reducing medical cost $[5,6]$. In recent years, China has gradually introduced this disease management model and applied it to the care and management of diabetes [7].

In this study, multidisciplinary cooperation was adopted to provide comprehensive and personalized services for patients. Meanwhile, according to the differences of different patients, individualized blood glucose management plans are formulated for patients, encouraging family members to participate in the blood glucose management of patients, and providing timely supervision and guidance. The study proves that the multi-disciplinary cooperation mediated by case managers is conducive to the comprehensive monitoring of maternal health during pregnancy, to develop the advantages of various disciplines, to make full use of medical resources, so as to improve the medical level and diagnosis and treatment effect, and to play a good role in blood glucose control during pregnancy.

\subsection{The Role of Case Management Model in Relieving the Pressure of Patients}

The results of this study showed that the case management did not significantly improve the rate of cesarean section, gestational week of delivery, postpartum hemorrhage, newborn birth weight, ratio of neonatal transfer, incidence of macrosomia, incidence of premature infants, incidence of neonatal hypoglycemia, neonatal asphyxia and other pregnancy outcomes. This is not consistent with the research results of quite a few researchers, which may be different from the fact that our hospital is a third-class first-class hospital, and routine management has been standardized in daily diagnosis and treatment activities, and abnormal conditions can be timely and effectively handled, so there is no big difference in other pregnancy outcomes [9-13]. On the other hand, it may be related to the number of samples in this study. In the future, the author will increase the sample size in the case management intervention study of gestational diabetes, so as to investigate and analyze the long-term care effect of case management through multi-center cooperation.

\section{Conclusion}

In conclusion, the case management model can enable patients to correctly grasp the relevant knowledge of diet, exercise and drug application of gestational diabetes management, effectively reduce the readmission rate of poor blood glucose control and the frequency of insulin use in patients, and improve the blood glucose control during pregnancy. As the improvement of living standards and the new Chinese birth policy, the increasing number of patients 
leads to the increasing demand for GDM quality management. The case management model provides patients with whole-course and continuous medical care, realizing the mutual benefit of medical treatment, patients and nursing. Therefore, the case management model is the inevitable trend of GDM management in the future.

\section{References}

[1] Mahrukh M A, Marium Z, Roha S M. Gestational diabetes mellitus and periodontal diseases: a newly well-established association [J]. Archives of Gynecology and Obstetrics, 2018, 298, 443-444.

[2] Riskin A, Garcia-Prats JA. Up to date: infant of a diabetic mother. In: Weisman LE, Wolfsdorf, JI, Kim MS, editors. http://www.uptodate.com/contents/infant-ofa-diabetic-mother? source=related_link-H714002. Accessed 25 Feb 2016.

[3] Jose M P, Anne M P, Jacomine d L, Peter M S, Anneke L F. Does the organisational model of dementia case management make a difference insatisfaction with case management and caregiver burden? An evaluation study [J]. BMC Geriatrics, $2016,16,65$.

[4] Li L, Zhang B, Ding Y. Practice and effect evaluation of gestational diabetes mellitus specialist practice model [J]. Chinese Journal of Nursing, 2017, 52 (05), 535-539.

[5] Lu Y. Semiotic clarity of Case Management Model and Notation (CMMN). China Digital Medicine, 2017, 12 (01), 86-88.

[6] Hongxin L. Quality control and management of electronic nursing records by the nursing team leader. Journal of Traditional Chinese Medicine Management, 2019, 27 (03), 66-67.
[7] Zi Z, Ting S, Lijuan Y, Yan W, Guomin S. Effect of case management on patients with type 2 diabetes mellitus: a meta-analysis. Chinese Nursing Research, 2016, 3 (2), 71-76.

[8] IDF Diabetes Atlas $8^{\text {th }}$ edition: http://www.diabetesatlas.org/resources/2017-atlas.html

[9] Judith P, Katherine S, Khalida I, Katherine H, Helen R, Angus F. Experiences of gestational diabetes and gestational diabetes care: a focus group and interview study. BMC Pregnancy and Childbirth, 2018, 18, 25.

[10] Zhu Y, Zhang C. Prevalence of gestational diabetes and risk of progression to type 2 diabetes: a global perspective. Curr Diab Rep. 2016, 16 (1), 7.

[11] Susan F, Grant C. A Systems Thinking Perspective of Medication Adherence for Patients with Diabetes Mellitus. Procedia Computer Science, 2019, 153, 218-224.

[12] Juan J G, Jean M C, Catherine D, Hasan I. Diabetes education and health insurance: How they affect the quality of care provided to people with type 1 diabetes in Latin America. Data from the International Diabetes Mellitus Practices Study (IDMPS). Diabetes Research and Clinical Practice, 2019, 147, 47-54.

[13] Mao F J. The influence of case management on gestational diabetes [J]. Health Research, 2016, 36 (04), 371-373.

[14] Mariana P S, Helena M G, Eduardo G L, Joice M V, Bianca N C, Roberto A d A C, Marilza V C R, Iracema d M P C. Diagnostic protocol for gestational diabetes mellitus (GDM) (IADPSG/ADA, 2011): influence on the occurrence of GDM and mild gestational hyperglycemia (MGH) and on the perinatal outcomes [J]. Diabetology \& Metabolic Syndrome, 2017, 9, 2 .

[15] Guangran Y, Timothy D D, Dongmei L. Effects of pre-gestational diabetes mellitus and gestational diabetes mellitus on macrosomia and birth defects in Upstate New York. Diabetes Research and Clinical Practice, 2019, 155, Article 107811. 